BNL-112626-2016-JA

\title{
Point-contact Andreev reflection spectroscopy on Bi2Se3 single crystals
}

C. R. Granstrom, I. Fridman, H.-C. Lei, C. Petrovic, and J. Y. T. Wei

Submitted to International Journal of Modern Physics B

May 2016

Condensed Matter Physics and Material Science Department

Brookhaven National Laboratory

\author{
U.S. Department of Energy \\ USDOE Office of Science (SC), \\ Basic Energy Sciences (BES) (SC-22)
}

Notice: This manuscript has been authored by employees of Brookhaven Science Associates, LLC under Contract No. DE-SC0012704 with the U.S. Department of Energy. The publisher by accepting the manuscript for publication acknowledges that the United States Government retains a non-exclusive, paid-up, irrevocable, world-wide license to publish or reproduce the published form of this manuscript, or allow others to do so, for United States Government purposes. 


\section{DISCLAIMER}

This report was prepared as an account of work sponsored by an agency of the United States Government. Neither the United States Government nor any agency thereof, nor any of their employees, nor any of their contractors, subcontractors, or their employees, makes any warranty, express or implied, or assumes any legal liability or responsibility for the accuracy, completeness, or any third party's use or the results of such use of any information, apparatus, product, or process disclosed, or represents that its use would not infringe privately owned rights. Reference herein to any specific commercial product, process, or service by trade name, trademark, manufacturer, or otherwise, does not necessarily constitute or imply its endorsement, recommendation, or favoring by the United States Government or any agency thereof or its contractors or subcontractors. The views and opinions of authors expressed herein do not necessarily state or reflect those of the United States Government or any agency thereof. 


\title{
Point-contact Andreev reflection spectroscopy on $\mathrm{Bi}_{2} \mathrm{Se}_{3}$ single crystals
}

\author{
C. R. Granstrom ${ }^{1}$, I. Fridman ${ }^{1}$, H.-C. Lei ${ }^{2}$, C. Petrovic ${ }^{2}$, and J. Y. T. Wei ${ }^{1,3}$ \\ 1 Department of Physics, University of Toronto, 60 St. George St., Toronto, \\ M5S1A7, Canada \\ 2 Condensed Matter Physics \& Materials Science Department, Brookhaven \\ National Laboratory, NY 11973, USA \\ 3 Canadian Institute for Advanced Research, Toronto, M5G1Z8, Canada
}

\begin{abstract}
To study how Andreev reflection (AR) occurs between a superconductor and a three-dimensional topological insulator (TI), we use superconducting $\mathrm{Nb}$ tips to perform point-contact $\mathrm{AR}$ spectroscopy at $4.2 \mathrm{~K}$ on as-grown single crystals of $\mathrm{Bi}_{2} \mathrm{Se}_{3}$. Scanning tunneling spectroscopy and scanning tunneling microscopy are also used to characterize the superconducting tip and both the doping level and surface condition of the TI sample. The point-contact measurements show clear spectral signatures of AR, as well as a depression of the zero-bias conductance with decreasing junction impedance. The latter observation can be attributed to interfacial Rashba spin-orbit coupling, and the presence of bulk bands at the Fermi level in our samples suggests that bulk states of $\mathrm{Bi}_{2} \mathrm{Se}_{3}$ are involved in the observed $\mathrm{AR}$.
\end{abstract}

\subsection{Introduction}

Three-dimensional topological insulators (TIs) are a novel class of materials where strong spin-orbit coupling causes bulk band inversion, resulting in surface states with Dirac dispersion and helical spin polarization that are topologically-protected against back-scattering [1,2]. Andreev reflection (AR) is the process that converts a normal-current to a super-current across a metal/superconductor interface. For conventional metals, AR has been extensively studied and is quite well understood [3-7]. For three-dimensional TIs, however, there have been no explicit experimental or theoretical studies of AR. Generally speaking, AR is known to be affected by Fermi-wavector mismatch [4], spin polarization [8-12], Rashba spin-orbit coupling [13-17], and Dirac dispersion [18]. Considering the unique attributes of three-dimensional TIs, it is therefore interesting to examine whether and how AR occurs onto topological surface states.

The preferred method for measuring AR is to place a sharp tip in ballistic contact with a sample, in a technique called point-contact Andreev reflection spectroscopy (PCARS), which has the following advantages. First, the small 
size of the tip/sample junction ensures local voltage drop. Second, spectral analysis is amenable to the Blonder-Tinkham-Klapwijk (BTK) theory, which was specifically developed for 1D microconstrictions [3]. Third, the pointcontact geometry minimizes local attenuation of the pair potential in the superconducting electrode $[7,19]$, thus simplifying spectral analysis. Fourth, the tip can be cleaned by in-situ field-emission. Lastly, many junctions can be made in a single experiment, to check for spectral reproducibility.

In this study, we perform PCARS on as-grown $\mathrm{Bi}_{2} \mathrm{Se}_{3}$ single crystals using superconducting Nb tips. Scanning tunneling spectroscopy (STS) and scanning tunneling microscopy are also used to characterize the superconducting tip and both the doping level and surface condition of the TI sample. These tunneling measurements show that our tip and sample are clean, and that the Fermi level of our as-grown $\mathrm{Bi}_{2} \mathrm{Se}_{3}$ crystals is in the bulk conduction band. The point-contact measurements show clear spectral signatures of $A R$, along with an asymmetric conductance background that can be attributed to the band structure of $\mathrm{Bi}_{2} \mathrm{Se}_{3}$. We also observe a depression of zero-bias conductance with decreasing junction impedance, which can be attributed to interfacial Rashba spin-orbit coupling. The presence of bulk bands at the Fermi level in our samples suggests that bulk states of $\mathrm{Bi}_{2} \mathrm{Se}_{3}$ are involved in the observed $\mathrm{AR}$.

\subsection{Experiment}

The $\mathrm{Bi}_{2} \mathrm{Se}_{3}$ single crystals used in this study were grown by the flux method. The as-grown crystals were n-type doped as a result of naturally occurring defects, which are common in the bismuth chalcogenides. PCARS and STS measurements were made using a home-built scanning tunneling microscope (STM) at $4.2 \mathrm{~K}$ in a ${ }^{4} \mathrm{He}$ dipper probe. For PCARS, differential conductance vs. bias voltage was measured using a four-terminal geometry with an AC resistance bridge. For STS, standard two-point AC lock-in technique was used. For PCARS, the STM approach method was used to to gently bring the tip and sample into contact without crashes. Namely, a piezo stepper motor with $\mathrm{a} \sim 10 \mathrm{~nm}$ step size was used to move the tip into tunneling range of the sample. The STM feedback was then disengaged, the four-terminal wiring engaged, and the tip gently brought into contact with the sample using the STM piezo. Nb tips of $99.9 \%$ purity were cut and cleaned in-situ through high-voltage field emission on a $\mathrm{Ag}$ film. The $\mathrm{Bi}_{2} \mathrm{Se}_{3}$ crystals were cleaved either in air or in-situ in $99.99 \%$ pure ${ }^{4} \mathrm{He}$ exchange gas. To check for spectral reproducibility, numerous $\mathrm{Nb}-\mathrm{Bi}_{2} \mathrm{Se}_{3}$ junctions were made on several crystals. Measurement accuracies of both the STS and PCARS setups were validated using $\mathrm{Nb}$ tips on Ag films and Au foil, respectively. 


\subsection{Results}

Fig. 1.1 shows typical PCARS data for a $\mathrm{Nb}$ tip on a $\mathrm{Bi}_{2} \mathrm{Se}_{3}$ crystal cleaved in air. The $d I / d V$ conductance spectra for various junction impedances are plotted vs. sample-to-tip bias voltage $V$, and staggered vertically for clarity. The spectrum for the highest junction impedance (80 ohms, bottom trace) shows a broad hump within $\pm 10 \mathrm{mV}$, indicating an $\mathrm{AR}$ conductance enhancement of $\sim 10 \%{ }^{4}$. As the tip is pushed into the sample, thereby decreasing the junction impedance to $5 \mathrm{ohms}$ (top trace), the zero-bias conductance dips and two peaks emerge. The peak separation is $\sim 4 \mathrm{mV}$, which can be compared with our STS data for a $\mathrm{Nb}$ tip on a Ag film, shown in Fig. 1.2(a). Although the superconducting gap of $\mathrm{Nb}$ is $\sim 1.5 \mathrm{meV}$ at $0 \mathrm{~K}$, spectral smearing due to finite quasiparticle lifetime and broadening of the Fermi-Dirac function at 4.2 $\mathrm{K}$ causes the coherence peaks in STS data to shift to $\sim \pm 2.5 \mathrm{mV}$. The clear AR peaks we observe in Fig. 1.1 are consistent with a clean tip as evidenced by the STS data in Fig. 1.2(a), and with a clean sample surface as shown by the atomically-resolved STM image in the inset of Fig. 1.2(b).

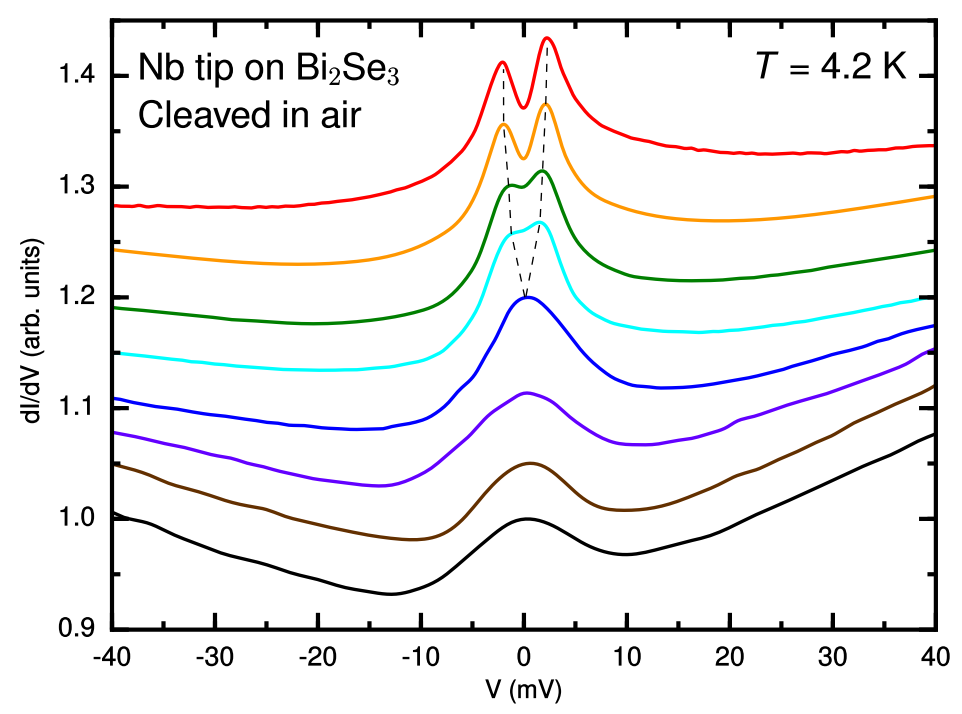

Fig. 1.1. PCARS data taken at $4.2 \mathrm{~K}$ with a $\mathrm{Nb}$ tip on air-cleaved $\mathrm{Bi}_{2} \mathrm{Se}_{3}$ as the tip is pushed into the sample. As the junction impedance decreases from $80 \mathrm{ohms}$ (bottom trace) to 5 ohms (top trace), the zero-bias conductance dips and two peaks emerge at $\pm 2 \mathrm{mV}$. Each trace is divided by its conductance at $-40 \mathrm{mV}$ and is shifted vertically for clarity.

\footnotetext{
${ }^{4}$ This conductance enhancement is defined as the zero-bias height of the AR hump relative to above-gap conductance, after normalizing the entire spectrum by a spectral background that is interpolated from outside the $\pm 15 \mathrm{mV}$ range.
} 

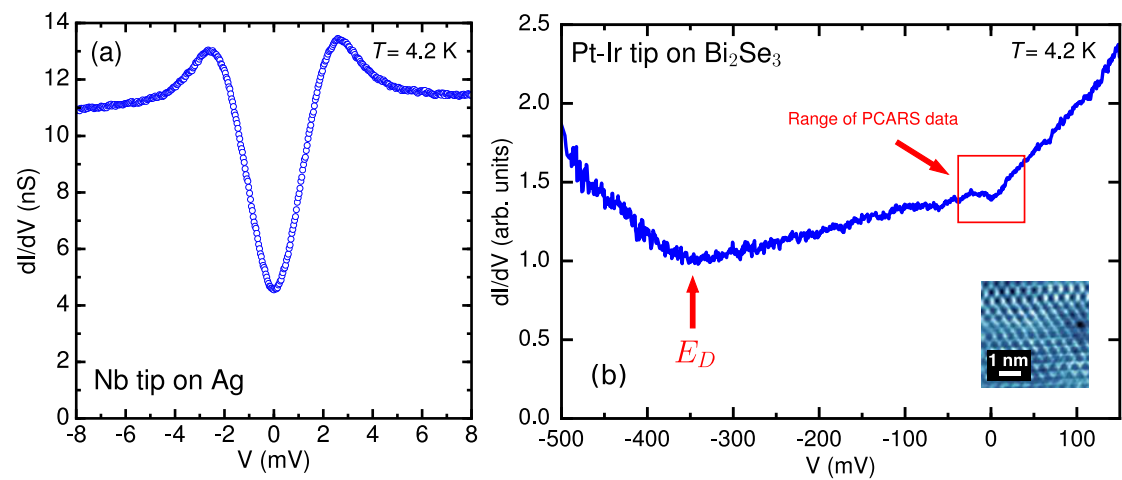

Fig. 1.2. (a) STS data for a $\mathrm{Nb}$ tip on a $\mathrm{Ag}$ film at $4.2 \mathrm{~K}$. The spectrum was taken with sample bias $V_{s}=-18 \mathrm{mV}$ and tunneling current $I_{t}=0.2 \mathrm{nA}$. (b) STS data taken on $\mathrm{Bi}_{2} \mathrm{Se}_{3}$ with a Pt-Ir tip at $4.2 \mathrm{~K}$, using $V_{s}=-80 \mathrm{mV}$ and $I_{t}=0.1 \mathrm{nA}$. As described in the text, the Fermi level is in the bulk conduction band (BCB), and the Dirac point is at $-350 \mathrm{mV}$. The inset shows an atomically-resolved STM image of a $\mathrm{Bi}_{2} \mathrm{Se}_{3}$ crystal with $V_{s}=-400 \mathrm{mV}$ and $I_{t}=2 \mathrm{nA}$.

In the standard BTK model, AR is not expected to produce any spectral asymmetry about zero bias. However, our PCARS data (Fig. 1.1) appear to be asymmetric, both in the AR peak heights and in the background conductance. To understand the origin of the peak-height asymmetry, we normalized each spectrum by dividing out the background conductance, after interpolating it from outside the $\pm 15 \mathrm{mV}$ range. This normalization procedure effectively symmetrized each spectrum (not shown), indicating that the peak-height asymmetry is due to the spectral background and thus is not intrinsic to the AR. The asymmetric spectral background is also seen in our STS data for $\mathrm{Bi}_{2} \mathrm{Se}_{3}$ (Fig. 1.2(b)). Its overall shape is consistent with previous STS data reported for $\mathrm{Bi}_{2} \mathrm{Se}_{3}$ single crystals [20], and with density-of-states calculations [21]. A more detailed comparison of our STS data with these prior studies indicate that, in our as-grown $\mathrm{Bi}_{2} \mathrm{Se}_{3}$ crystals, the Fermi level is in the bulk conduction band $(\mathrm{BCB})$ and the Dirac point $\left(E_{D}\right)$ is $\sim 350 \mathrm{mV}$ below the Fermi level, with no bulk bands lying at the same energy as $E_{D}$ [22].

Fig. 1.3 shows PCARS data taken on a $\mathrm{Bi}_{2} \mathrm{Se}_{3}$ crystal that was cleaved in-situ in $99.99 \%$ pure ${ }^{4} \mathrm{He}$ gas. In these spectra, as the junction impedance is decreased from 20 ohms to 10 ohms, a hump structure also splits into two peaks, but farther apart than the data for the air-cleaved crystal (Fig. 1.1). Additionally, the AR conductance enhancement here $(\sim 2 \%$ for the bottom trace in Fig. 1.3) is weaker than for the air-cleaved crystal $(\sim 10 \%$ for the bottom trace in Fig. 1.1). The differences between these two sets of data can be understood in terms of the "spreading" resistance resulting from the wiring configuration. Namely, unlike ex-situ cleaved crystals where the sample leads are on the crystal surface, in-situ cleaving requires the sample leads to be attached to the sides of the crystal, thus forcing the current to 
flow along the $c$-axis of the crystal. This is illustrated in the inset of Fig. 1.3. Since the $c$-axis resistivity of $\mathrm{Bi}_{2} \mathrm{Se}_{3}$ is $\sim 15$ times larger than its $a b$-plane resistivity [23], the four-terminal resistance can be effectively "spread" outside the point contact and into the sample. Such spreading resistance tends to lower and to broaden the measured AR characteristics [24,25], thus explaining the spectral differences between our ${ }^{4} \mathrm{He}$-cleaved and air-cleaved crystals. Here, it is worth noting a peculiar spectral feature in the ${ }^{4} \mathrm{He}$-cleaved case, namely the dips that evolve into humps at $\sim \pm 12 \mathrm{mV}$ as junction impedance decreases. The origin of this feature is not yet known, but its apparent correlation with the AR peak splitting suggests that it is related to AR.

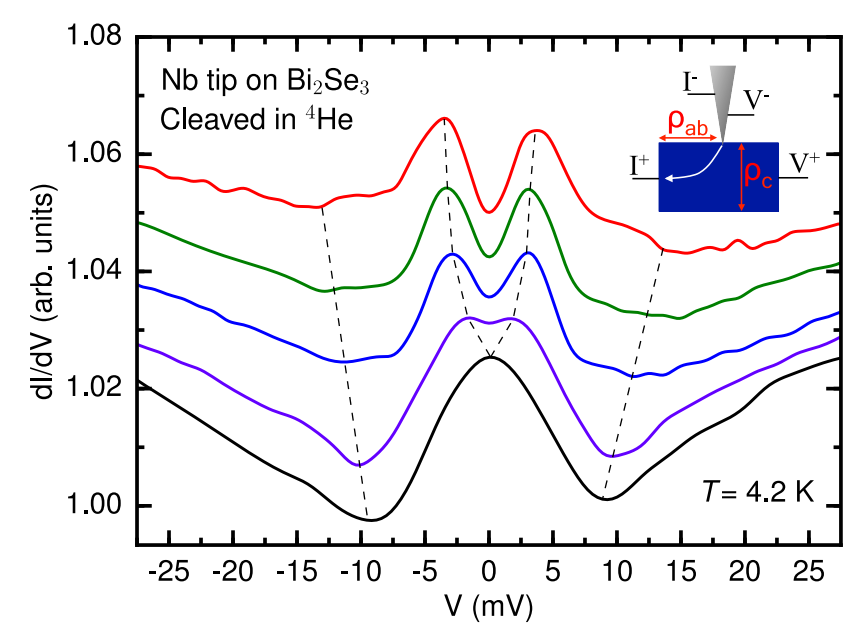

Fig. 1.3. PCARS data taken at $4.2 \mathrm{~K}$ with a $\mathrm{Nb}$ tip on $\mathrm{Bi}_{2} \mathrm{Se}_{3}$ cleaved in $99.99 \%$ pure ${ }^{4} \mathrm{He}$ exchange gas, as the tip is pushed into the sample. As the junction impedance decreases from 20 ohms (bottom trace) to $10 \mathrm{ohms}$ (top trace), a broad hump splits into two peaks separated by $7 \mathrm{mV}$. The AR conductance enhancement is weaker here than for $\mathrm{Bi}_{2} \mathrm{Se}_{3}$ cleaved in air. Also visible are dips that evolve into humps at \pm $12 \mathrm{mV}$. Each trace is divided by its conductance at $-25 \mathrm{mV}$ and is shifted vertically for clarity. The inset shows the contact configuration required for in-situ cleaving, whereby the current has to flow along the higher resistance $c$-axis.

\subsection{Discussion}

In our PCARS data, the hump-to-peaks spectral evolution versus decreasing junction impedance may be attributed to interfacial Rashba spin-orbit coupling (RSOC). In a non-topological context, RSOC has already been shown to depress AR at low energies [13-16]. A mechanism that may enhance this AR depression is band bending, which would strengthen the RSOC by inducing an electric field at the interface. Band bending can occur at an interface 
between two materials whenever there is a large difference in their Fermi energies, such as between a metal and a semiconductor [26]. In our case of an interface between metallic $\mathrm{Nb}$ and semimetallic $\mathrm{Bi}_{2} \mathrm{Se}_{3}$, a decrease in the junction impedance could increase the band bending, thereby strengthening the field-induced RSOC to enhance the AR depression. It is worth noting that $\mathrm{Bi}_{2} \mathrm{Se}_{3}$ is susceptible to band bending via surface adsorbates, as has been observed by ARPES [27-30] on samples deliberately exposed to water vapor.

It is likely that our PCARS data is influenced by topologically trivial bulk states present at the crystal surface, since our STS data indicate that the Fermi level crosses the BCB in our as-grown crystals. A natural question is, to what extent is the Andreev reflection occurring onto the topological surface states (TSS)? To answer this question, the dimensionality difference between the TSS and the bulk bands must be taken into account, as two- and threedimensional extensions of the BTK theory have shown that AR is affected by Fermi-surface dimensionality $[31,32]$. Other likely important factors are the spin-momentum locking and Dirac dispersion of the TSS. For instance, the former factor may enhance AR via suppression of back-scattering. Further refinements of the BTK theory would be needed to elucidate this possibility.

\subsection{Summary}

In an effort to understand how AR occurs between a superconductor and a three-dimensional topological insulator (TI), we have performed PCARS using superconducting $\mathrm{Nb}$ tips on as-grown single crystals of $\mathrm{Bi}_{2} \mathrm{Se}_{3}$. STS and scanning tunneling microscopy were also used to characterize the superconducting tip and both the doping level and surface condition of the TI sample. These tunneling measurements show that our tip and sample are clean, and that the Fermi level of our as-grown $\mathrm{Bi}_{2} \mathrm{Se}_{3}$ crystals is in the bulk conduction band. Our PCARS data show clear spectral signatures of AR, along with an asymmetric conductance background that can be attributed to the band structure of $\mathrm{Bi}_{2} \mathrm{Se}_{3}$. We also observe a depression of zero-bias conductance with decreasing junction impedance, which can be attributed to interfacial Rashba spin-orbit coupling. The presence of bulk bands at the Fermi level indicates that both bulk and surface states are involved in the observed AR, with the dimensionality difference between these states determining their relative contributions to the AR. Other important factors to consider are the spin-momentum locking and Dirac dispersion of the TSS. Further measurements, along with refinements of the BTK theory taking into account the unique attributes of TIs, are necessary to clarify these issues.

\section{References}

1. M. Z. Hasan, C. L. Kane, Rev. Mod. Phys. 82, 3045 (2010). 
2. X.-L. Qi, S.-C. Zhang, Rev. Mod. Phys. 83, 1057 (2011).

3. G. E. Blonder, M. Tinkham, T. M. Klapwijk, Phys. Rev. B 25, 4515 (1982).

4. G. E. Blonder, M. Tinkham, Phys. Rev. B 27, 112 (1983).

5. B. Pannetier, H. Courtois, J. Low Temp. Phys. 118(5-6), 599 (2000).

6. T. Klapwijk, J. Supercond. 17(5), 593 (2004).

7. D. Daghero, R. S. Gonnelli, Supercond. Sci. Technol. 23(4), 043001 (2010).

8. M. J. M. de Jong, C. W. J. Beenakker, Phys. Rev. Lett. 74, 1657 (1995).

9. R. J. Soulen, et al., Science 282(5386), 85 (1998).

10. Y. Ji, et al., Phys. Rev. Lett. 86, 5585 (2001).

11. I. Žutić, J. Fabian, S. Das Sarma, Rev. Mod. Phys. 76, 323 (2004).

12. S. K. Upadhyay, A. Palanisami, R. N. Louie, R. A. Buhrman, Phys. Rev. Lett. 81, 3247 (1998).

13. T. Yokoyama, Y. Tanaka, J. Inoue, Phys. Rev. B 74, 035318 (2006).

14. S. Wu, K. V. Samokhin, Phys. Rev. B 82, 184501 (2010).

15. S. Wu, K. V. Samokhin, Phys. Rev. B 81, 214506 (2010).

16. A. Kononov, G. Biasiol, L. Sorba, E. Deviatov, JETP Lett. 98(7), 421 (2013).

17. P. Högl, A. Matos-Abiague, I. Žutić, J. Fabian, Phys. Rev. Lett. 115, 116601 (2015).

18. C. W. J. Beenakker, Phys. Rev. Lett. 97, 067007 (2006).

19. G. Deutscher, Rev. Mod. Phys. 77, 109 (2005).

20. T. Hanaguri, K. Igarashi, M. Kawamura, H. Takagi, T. Sasagawa, Phys. Rev. B 82, 081305 (2010).

21. Y. Zhao, Y. Hu, L. Liu, Y. Zhu, H. Guo, Nano Lett. 11(5), 2088 (2011), PMID: 21495641.

22. Y. Xia, et al., Nat Phys 5(6), 398 (2009).

23. W. H. Jiao, et al., AIP Advances 2(2), 022148 (2012).

24. T. Y. Chen, S. X. Huang, C. L. Chien, Phys. Rev. B 81, 214444 (2010).

25. B. Nadgorny, Handbook of Spin Transport and Magnetism. Taylor and Francis, New York (2011).

26. H. Lüth, Solid Surfaces, Interfaces, and Thin Films. Springer, 5th edn. (2010).

27. M. Bianchi, et al., Nat Commun 1, 128 (2010).

28. H. M. Benia, C. Lin, K. Kern, C. R. Ast, Phys. Rev. Lett. 107, 177602 (2011).

29. P. D. C. King, et al., Phys. Rev. Lett. 107, 096802 (2011).

30. Z.-H. Zhu, et al., Phys. Rev. Lett. 107, 186405 (2011).

31. D. Daghero, M. Tortello, G. A. Ummarino, R. S. Gonnelli, Reports on Progress in Physics 74(12), 124509 (2011).

32. D. Daghero, M. Tortello, P. Pecchio, V. A. Stepanov, R. S. Gonnelli, Low Temperature Physics 39(3), 199 (2013). 